\title{
A Missed Screening Opportunity for Diabetes
}

\author{
Eva Tseng, MD, MPH and Nisa M. Maruthur, MD, MHS ${ }^{1,2,3}$
}

'Division of General Internal Medicine, The Johns Hopkins University School of Medicine, Baltimore, MD, USA; ${ }^{2}$ Department of Epidemiology, The Johns Hopkins University Bloomberg School of Public Health, Baltimore, MD, USA; ${ }^{3}$ Welch Center for Prevention, Epidemiology, and Clinical Research, The Johns Hopkins University, Baltimore, MD, USA.

J Gen Intern Med 32(4):373-4

DOI: $10.1007 / \mathrm{s} 11606-016-3970-1$

(c) Society of General Internal Medicine 2016

$\mathrm{D}$ iabetes disproportionately affects members of racial and ethnic minority groups in the U.S. ${ }^{1}$ Compared to nonHispanic whites (11.3\%), the age-standardized prevalence of total diabetes is higher for Asian Americans (20.6\%), Hispanic Americans (22.6\%), and non-Hispanic blacks (21.8\%). ${ }^{2}$ Given the burden of diabetes within these specific populations, the U.S. Preventive Services Task Force (USPSTF) revised its recommendations in 2014 to include screening for type 2 diabetes among certain racial and ethnic minority groups (African Americans, American Indians or Alaska Natives, Asians, Hispanics, and Native Hawaiians or other Pacific Islanders). ${ }^{1}$ Despite these recommendations, there is evidence that the prevalence of undiagnosed diabetes is higher among Asian Americans (50.9\%) and Hispanic Americans (49.0\%) compared to all other racial and ethnic minority groups. ${ }^{2}$ Although Asian Americans represent only 4.8\% of the U.S. population, they are the fastest-growing racial group in the country, based on the 2010 Census. $^{3}$

In this issue of JGIM, Tung et al. ${ }^{4}$ examine racial and ethnic disparities in diabetes screening between Asian Americans and other adults using an analysis of pooled cross-sectional data from the 2012-2014 Behavioral Risk Factor Surveillance System (BRFSS). ${ }^{4}$ The authors estimated the weighted proportions of adults in each racial and ethnic group who selfreported receipt of diabetes screening among the total population who were eligible for diabetes screening based on the American Diabetes Association guidelines (age $\geq 45$ years or age $<45$ years with body mass index $\geq 25 \mathrm{~kg} / \mathrm{m}^{2}$ ).

They found that Asian Americans were least likely to receive recommended diabetes screening compared to nonHispanic whites $(47.1 \%$ vs. $59.2 \%$, weighted; $\mathrm{AOR}=0.66$; 95\% CI: 0.60 to 0.73 ), adjusting for age, gender, education, residence in a metropolitan area and healthcare access. In contrast, non-Hispanic blacks and Hispanic/Latinos were more likely to receive recommended diabetes screening compared to non-Hispanic whites. The authors also found that these racial and ethnic disparities in diabetes screening among Asian Americans persisted even when they stratified their

Published online January 3, 2017 results by age and body mass index. Asian Americans aged $\geq 45$ years with $\mathrm{BMI} \geq 30 \mathrm{~kg} / \mathrm{m}^{2}$ had the largest disparity in receiving recommended diabetes screening compared to their non-Hispanic obese white counterparts (AOR $=0.56 ; 95 \% \mathrm{CI}$ : 0.39 to 0.81 ). Furthermore, this disparity persisted when they examined the subgroup of individuals aged $\geq 50$ years who had ever received colorectal cancer and/or breast cancer screening. ${ }^{4}$

The major strength of this study is that it provides nationally representative data on diabetes screening in a multiracial sample. Study limitations include the use of self-reporting of diabetes screening, which to our knowledge has not been validated. Also, given that diabetes screening may be done on "routine" lab testing, patients may not be aware that they are being screened; therefore, screening for diabetes could be underreported, although this underreporting is unlikely to be differential by race. A general weakness of the BRFSS is that it does not delineate between different Asian American subgroups, and there is evidence of significant ethnic variation in diabetes prevalence. ${ }^{5}$ Additionally, BRFSS is conducted primarily in English, although a Spanish-language instrument is available. Level of English proficiency is not assessed in the survey.

There are several take-away points from this study. One, to successfully curb the diabetes epidemic, we must increase both provider and patient awareness of these differences in diabetes risk among racial and ethnic groups. Data from the National Health Interview Survey demonstrated that the prevalence of diabetes is highest among Asian Indians and Filipinos and lowest among Koreans, Vietnamese and Chinese. $^{5}$ Furthermore, there is significant heterogeneity in the prevalence of overweight and obesity among Asian Americans, ${ }^{5}$ which may explain some of the ethnic variation in diabetes risk.

Second, we need to provide consistent guidelines for clinicians and increase awareness of these guidelines. In 2015, the American Diabetes Association changed its guidelines to recommend screening for diabetes among Asian Americans with a lower BMI cutoff of $23 \mathrm{~kg} / \mathrm{m}^{2.6}$ The USPSTF also issued recommendations in 2015 to screen for abnormal blood glucose in adults aged 40 to 70 years who are overweight and obese, and noted that "certain racial/ethnic groups may be at increased risk for diabetes at a lower body mass index" but did not specify cutoffs. ${ }^{7}$ Additional efforts to increase knowledge around these specific screening guidelines, such as the "Screen 
at 23" campaign promoted by the National Council of Asian Pacific Islander Physicians, are critical. ${ }^{8}$

Third, research on the diabetes risk among Asian Americans has been limited. Only five prospective cohort studies have reported on diabetes incidence among Asians (in the U.S. and Canada) from 2009 to 2013. ${ }^{9}$ Furthermore, while Asian Americans may be included in the survey population, some national surveys like BRFSS exclude Asian Americans in many of their data reports and publications. ${ }^{10}$ This study by Tung et al. is a much needed addition to the sparse national epidemiologic data on Asian Americans.

In conclusion, diabetes disproportionately affects members of racial and ethnic minority groups in the U.S. The disparity in diabetes screening among Asian Americans is underrecognized and understudied. The study by Tung et al. provides valuable preliminary data and lays the foundation for further studies on this fastest-growing racial group in America.

Corresponding Author: Eva Tseng, MD, MPH; Division of General Internal MedicineThe Johns Hopkins University School of Medicine, Baltimore, MD, USA (e-mail: etseng3@jhmi.edu).

\section{Compliance with Ethical Standards:}

Conflict of Interest: The authors declare that they do not have a conflict of interest.

\section{REFERENCES}

1. Centers for Disease Control and Prevention. Diabetes Report Card 2014. Atlanta, GA: Centers for Disease Control and Prevention, US Dept. of Health and Human Services; 2015.

2. Menke A, Casagrande S, Geiss L, Cowie CC. Prevalence of and trends in diabetes among adults in the United States, 1988-2012. JAMA. 2015;314(10): 1021-1029.

3. Hoeffel EM, Rastogi S, Kim MO, Shahid H. The Asian Population: 2010. Washington, D.C.: U.S. Census Bureau; 2012.

4. Tung EL, Baig AA, Huang ES, Laiteerapong N, Chua KP. Racial and Ethnic Disparities in Diabetes Screening between Asian Americans and Other Adults: BRFSS 2012-2014. J Gen Intern Med. 2016. doi:10. 1007/s11606-016-3913-x.

5. Golden SH, Brown A, Cauley JA, et al. Health disparities in endocrine disorders: biological, clinical and nonclinical factors-an Endocrine Society Scientific Statement. J Clin Endocrinol Metab. 2012;97(9):E1579-E1639.

6. Professional Practice Committee of the American Diabetes Association. Standards of medical care in diabetes-2015. Diabetes Care. 2015;38(Suppl. 1):S1-S2.

7. Siu AL, U.S. Preventive Services Task Force. Screening for abnormal blood glucose and type 2 diabetes mellitus: U.S. Preventive Services Task Force Recommendation Statement. Ann Intern Med. 2015;163(11):861868.

8. Screen at 23. Screen at 23. http://screenat23.org/about/. Accessed November 6, 2016

9. Hsu WC, Araneta MR, Kanaya AM, Chiang JL, Fujimoto W. BMI cut points to identify at-risk Asian Americans for type 2 diabetes screening. Diabetes Care. 2015;38(1):150-158.

10. Holland AT, Palaniappan LP. Problems with the collection and interpretation of Asian-American health data: omission, aggregation and extrapolation. Ann Epidemiol. 2012;22(6):397-405. 